\title{
Programming a Cochlear Implant for Tinnitus Suppression
}

\author{
Ann Perreau ${ }^{1,2}$ Richard Tyler ${ }^{2,3}$ Patricia C. Mancini²,4
}

${ }^{1}$ Department of Communication Sciences and Disorders, Augustana College, Rock Island, IL

${ }^{2}$ Department of Otolaryngology, Head and Neck Surgery, University of lowa, lowa City, IA

${ }^{3}$ Communication Sciences and Disorders, University of lowa, lowa City, IA

${ }^{4}$ Department of Speech-Language Pathology and Audiology, Universidade Federal de Minas Gerais, Belo Horizonte, Brazil

J Am Acad Audiol 2020;31:302-308.
Address for correspondence Ann Perreau, Department of Communication Sciences and Disorders, Augustana College, Rock Island, IL 61201 (e-mail: annperreau@augustana.edu).

\begin{abstract}
Keywords

- cochlear implants

- tinnitus
\end{abstract}

Electrical stimulation of the cochlea to treat tinnitus has been explored for decades. The effect of cochlear implantation on tinnitus varies significantly, ranging from $54 \%$ to $86 \%$ of adults reporting partial to complete tinnitus suppression (e.g., Souliere et al, $1992^{28}$; Kou et al, $1994^{29}$; Tyler, $1994^{29}$; Ito, $1997^{14}$; Mo et al, $2002^{19}$ ). Despite these positive results, tinnitus will remain bothersome for some patients (2-9\%; Tyler and Kelsay, $1990^{30}$; Hazell et al, $1995^{11}$; Mo et al, $2002^{19}$ ) receiving a cochlear implant (CI). The goals of electrical stimulation for tinnitus suppression are different than for improving speech perception, i.e., to provide 'silence' versus maximize audibility and speech understanding. Therefore, it is likely that the optimal stimulation parameters for tinnitus suppression will differ from those for speech perception (Zeng et al, 2011 ${ }^{35}$ ). There are many reports on the effect of programming parameters on speech perception of CI patients (e.g., Plant et al, $2002^{21}$; Koch et al, 2004 ${ }^{26}$; Balkany et al, 200733; Firszt et al, $\left.2009^{6}\right)$. These CI programming parameters include signal processing/coding strategy (e.g., ACE, FSP, and HiRes), stimulation mode of electrodes (e.g., bipolar versus monopolar), stimulation rate (e.g., $125-5100 \mathrm{~Hz}$ ), input frequency to electrode allocation table, stimulation channels (e.g., 12 to 24), threshold levels (minimum level producing audible sound, abbreviated T), and most comfortable levels (MCLs) (maximum output level, abbreviated MCL, M, or C).

However, few large-scale studies have investigated the most salient programming parameters for tinnitus suppression in CI patients who remain bothered by their tinnitus after implantation. Dauman and Tyler $(1993)^{4}$ were the first to vary the current level of the electrical stimulation and document the effects on tinnitus loudness for two $\mathrm{CI}$ patients. Rubinstein et al $(2003)^{26}$ developed a high-rate conditioner stimulus of $5000 \mathrm{~Hz}$ in an attempt to restore spontaneous neural activity without producing audible
Copyright (C) 2020 by the American Academy of Audiology. All rights reserved. Thieme Medical Publishers, Inc., 333 Seventh Avenue, New York, NY 10001, USA. Tel: +1(212) 760-0888
DOI https://doi.org/ 10.3766/jaaa.18086 ISSN 1050-0545. 
sound. Tinnitus was successfully suppressed in $30-50 \%$ of the participants using transtympanic electrodes (11 adults) and Cls (three adults), and there was minimal detection of the conditioner stimulus. In three participants who experienced tinnitus suppression, lowering the stimulation rate and current level of the conditioner stimulus provided less tinnitus reduction than a high-rate conditioner stimulus. By comparison, other researchers have reported tinnitus suppression with low stimulation rates (e.g., $<100 \mathrm{~Hz}$; Zeng et al, 2011). ${ }^{35}$

More recently, Liu et al $(2016)^{18}$ assessed the effect of programming adjustments for $\mathrm{CI}$ patients with tinnitus. They hypothesized that electrode impedance, an indicator of changes in the $\mathrm{Cl}$ over time, would decrease following programming adjustments and may alter tinnitus symptoms. The patients were grouped by tinnitus onset: (a) 108 with tinnitus preimplantation, (b) 88 with tinnitus after surgery, but before initial activation, and (c) 44 with tinnitus one year postimplantation. Patients in each group were randomly assigned to receive regular programming at $4,6,8$, and 12 weeks postimplantation or programming at four weeks postimplantation (i.e., at initial activation) with no subsequent programming. Results revealed that electrode impedance was significantly lower in the programming subgroups with pre- and postoperative tinnitus. In addition, a greater, more immediate reduction was reported in tinnitus handicap for the groups who received regular programming (Liu et al, $2016^{18}$ ). They concluded that regular CI programming lowered electrode impedance, which effectively suppressed tinnitus in the patients who experienced it preoperatively, postoperatively before initial activation, and postoperatively by at least one year after initial activation. Programming adjustments were limited to $T$ and $\mathrm{C}$ levels with other parameters set consistently across participants: ACE strategy at $900 \mathrm{~Hz}$ stimulation rate with 22 channels and eight maxima. Finally, previous studies found no significant correlation between $\mathrm{CI}$ device type to tinnitus awareness, tinnitus distress, or tinnitus handicap scores (Quaranta et al, 2008; ${ }^{24}$ Andersson et al, 2009; ${ }^{1}$ Gomersall et al, $\left.2019^{10}\right)$. In summary, studies indicate that regular programming adjustments, different programming parameters, and various $\mathrm{CI}$ devices can result in tinnitus suppression, although an exploration of the most salient programming parameters for tinnitus suppression is needed.

The purpose of this article was to review the available CI programming parameters for tinnitus suppression and to consider possible clinical research designs for selecting the optimal programming parameters for clinical applications. This information may be helpful to audiologists who provide tinnitus management to their $\mathrm{Cl}$ patients and has direct implications in the development of therapeutic interventions for $\mathrm{Cl}$ patients with tinnitus.

\section{Programming Parameters for Tinnitus Suppression}

\section{Signal Coding Strategy}

Few studies have directly evaluated the impact of signal coding strategies on tinnitus. Quaranta et al $(2008)^{24}$ found that more sophisticated and higher rate strategies (ACE,
HiRes, and CIS) were more effective than slower strategies (SAS and SPEAK) in suppressing tinnitus for $41 \mathrm{CI}$ patients. Significant differences emerged between the signal coding strategies when the $\mathrm{CI}$ was on but not when the $\mathrm{CI}$ was off. However, the effect on individual patients was not considered because only group data were presented. A review of 32 studies (Quaranta et al, $2004^{23}$ ) found that multichannel CIs were more effective for tinnitus suppression than singlechannel Cls. As suggested by Quaranta et al $(2008)^{24}$, more complex stimulation may provide additional temporal information to stimulate the auditory nerve fibers and the central auditory pathway and result in tinnitus suppression. Greater auditory input from the $\mathrm{CI}$ has the potential to mask tinnitus, effectively changing the neural representation within the brain and allowing patients to refocus their attention away from tinnitus. Therefore, although few studies have evaluated signal-coding strategies for tinnitus suppression, the literature suggests that more sophisticated and higher rate strategies appear to be more effective.

\section{Rate of Stimulation}

Low and high rates of electrical stimulation are effective in suppressing tinnitus in CI patients, although the specific results vary across studies. Dauman and Tyler $(1993)^{4}$ reported that a stimulation rate of $125 \mathrm{~Hz}$ at $20 \%$ of the stimulus loudness was effective in suppressing tinnitus for two CI patients. Furthermore, they found that less electrical current was needed at $125 \mathrm{~Hz}$ to suppress the tinnitus compared with rates of 80, 250, and $500 \mathrm{~Hz}$. Hazell et al $(1989)^{12}$ demonstrated that tinnitus could be suppressed with a low, $100-\mathrm{Hz}$ sinusoid in six CI patients. Zeng et al $(2011)^{35}$ reported that low-rate stimulation $(<100 \mathrm{~Hz})$ was effective in suppressing tinnitus for one $\mathrm{CI}$ patient with unilateral hearing loss and severe tinnitus. In that study, they investigated various high-rate stimulation rates and patterns, but complete tinnitus suppression resulted when using a low, $100-\mathrm{Hz}$ stimulation rate. Early reports also suggested that the optimal stimulation rate for tinnitus suppression might be different for different patients (Kuk et al, $1989^{17}$ ). Electrical stimulation using rates from 62 to $8000 \mathrm{~Hz}$ was effective in reducing tinnitus for 5 of 10 participants using a ball electrode on the eardrum.

Rubinstein et al $(2003)^{26}$ developed a novel high-rate conditioner stimulus (i.e., $5000 \mathrm{~Hz}$ ) presented along with the $\mathrm{Cl}$ signal to suppress tinnitus in patients using both intracochlear and transtympanic electrodes (Rubinstein and Tyler, $2004^{26}$ ). In the development of the conditioner stimulus, multiple rates of stimulation were compared, but a high-rate stimulus was found to be themost effective in suppressing tinnitus when presented in conjunctionwith the signal fromthe $\mathrm{Cl}$ (Rubinstein et al, $2003^{26}$ ). Although these studies were conducted with a small number of participants, the results fromindividual participants indicate that audiologists should try programs with low- and high-stimulation rates for their $\mathrm{Cl}$ patients with bothersome tinnitus.

Several findings resulted from these studies on rate of stimulation for tinnitus suppression. First, the length of electrical stimulation for tinnitus suppression ranged from 3 (Zeng et al, $2011^{35}$ ) to 15 minutes (Rubinstein et al, $2003^{26}$ ), 
suggesting that a slow, central adaptation process may be involved for some patients. Once electrical stimulation was turned off, the effects on tinnitus varied significantly. In some patients, a 'rebound' of tinnitus occurred that was worse than baseline (Zeng et al, $2011^{35}$ ). For other patients, residual inhibition of the tinnitus lasted from 45 minutes to three days (Rubinstein et al, $2003^{26}$ ). Across studies, it was observed that tinnitus suppression diminished steadily over time with continuous stimulation. When this occurred, changes to the stimulus parameters (i.e., increasing current level) were required to suppress tinnitus successfully. Finally, some studies (Rubinstein et al, $2003 ;{ }^{26}$ Zeng et al, $2011^{35}$ ) used custom research platforms to deliver the electrical stimulation due to the limitations in the stimulus parameters within the manufacturer's sound processors and software. It is anticipated that more options for programming CIs for tinnitus suppression will be available from $\mathrm{CI}$ manufacturers as more research is conducted with a greater number of $\mathrm{CI}$ patients with tinnitus.

\section{Electrode Location and Number}

Studies have found that the electrode location affects the current that is needed to suppress tinnitus. In addition, the optimal location for tinnitus suppression appears to vary from patient to patient, although these results are limited to reports from single participants. Dauman and Tyler (1993) ${ }^{4}$ reported that the middle electrodes required less current to suppress tinnitus compared with more apical or basal electrodes for two patients using intracochlear devices. Specifically, data from one tinnitus patient with only 11 active electrodes (only electrodes 21 to 11 were available because of a severe labyrinthine fracture) revealed that the electrodes positioned in the middle of the array (e.g., 13 and 15) required considerably less current to suppress tinnitus than those at the end (e.g., 21). Other researchers suggested that stimulation using the entire electrode array was more effective in reducing tinnitus compared with stimulation of only the basal electrodes (Punte et al, $\left.2013^{22}\right)$. Furthermore, Zeng et al $(2011)^{35}$ found that the four most apical electrodes with a low-rate of stimulation (20$100 \mathrm{~Hz}$ ) were more effective in suppressing high-pitched tinnitus than the basal electrodes. It has been suggested that tinnitus suppression using the low-rate stimulation in the apex of the cochlea rather than the base indicates that a certain place mechanism might be related to restored inhibition within the auditory system (Zeng et al, $2011^{35}$ ).

In an attempt to determine the electrodes that are most effective for tinnitus suppression, some researchers (Rubinstein et al, 2003; ${ }^{26}$ Rubinstein and Tyler, 2004 ${ }^{25}$ ) have matched electrodes to the patient's tinnitus pitch. Using this approach, Rubinstein et al $(2003)^{26}$ found that tinnitus suppression occurred with the pitch-matched electrodes of 7 and 14 located in the middle of the array. In addition, Rubinstein and Tyler $(2004)^{25}$ reported on a case study of a patient with unilateral hearing loss and severe tinnitus due to Meniere's disease who received a CI. One month postactivation, the patient matched the pitch of her tinnitus to an apical electrode, and the high-rate conditioner stimulus was effective in suppressing her low-frequency tinnitus. Results across studies are variable with regard to the optimal electrode location for tinnitus suppression likely because of the small number of participants included in each study. With this limitation in mind, preliminary results indicate that measuring the pitch of the patient's tinnitus might be a necessary step for tinnitus suppression via a CI.

In addition, decreasing or increasing the number of electrodes in the patient's program may alter the tinnitus percept. First, many studies have reported that speech perception (e.g., vowel and consonant perception) improves as the number of electrodes is increased up to about eight electrodes, and adding additional electrodes above 8 to 12 produces less improvement (Fishman et al, $1997 ;^{7}$ Dorman et al, 2000; ${ }^{5}$ Friesen et al, $2001^{8}$ ). More recently, SchvartzLeyzac et al (2017) $)^{27}$ found that performance improved for more challenging speech tests when 20 electrodes were activated compared with only eight electrodes. Consistent with these findings, one study found that some bilateral $\mathrm{CI}$ patients showed a gradual degradation in performance on more complex tasks such as sound localization and speechin-noise perceptionwhen the number of electrodeswere gradually reduced from 20 to 1 (Perreau et al, 2010 20 ). Multiple studies have observed large individual differences in performance when the number of $\mathrm{CI}$ electrodes is reduced (Fishman et al, $1997 ;^{7}$ Frijns et al, $2003 ;^{9}$ Perreau et al, $2010 ;^{20}$ Schvartz-Leyzac et al, $2017^{27}$ ). In addition,many factors may influence performance with reduced electrodes such as the signal coding strategy (ACE versus CIS), channel interaction, and stimulation mode (tripolar versus monopolar). Thus, a more conservative approach is advocated when deactivating electrodes tomaintain optimal CI performance.

From our informal discussions with audiologists, many audiologists will eliminate $\mathrm{Cl}$ electrodes if the patient reports that high- or low-pitched sounds interfere with or worsen their tinnitus. For example, if stimulation from a basal electrode exacerbates a patient's tinnitus, then this electrode might be eliminated from the patient's program. As a result, the frequency allocation table is changed because those frequencies are assigned to the remaining electrodes, which might stimulate different neural fibers. Although it may be detrimental for speech perception when spectral resolution is decreased (Schvartz-Leyzac et al, $2017^{27}$ ), eliminating certain electrodes may be helpful for some patients to suppress their tinnitus. Conversely, for some CI patients with bothersome tinnitus, adding electrodes back into the CI program might be indicated if they were deactivated because of performance and sound quality issues. Reactivating certain electrodes may provide a low-level background sound to mask tinnitus and change the neural excitation patterns that produce tinnitus (Tyler et al, 2008a $\mathrm{a}^{31}$ ). Regardless, speech recognition performance should be monitored when changing the number of electrodes in a $\mathrm{CI}$ patient's program or when using low-level background sound to mask tinnitus in CI patients (Rubinstein et al, 2003; ${ }^{26}$ Tyler et al, $2015^{33}$ ). If the audiologist takes a more conservative approach to programming, adjusting $\mathrm{T}$ and $\mathrm{C}$ levels as a first attempt to maintain speech perception would be advised before eliminating $\mathrm{CI}$ electrodes. 


\section{Threshold (T-Levels)}

Based on our discussions with audiologists and CI manufacturers, decreasing T-levels have been recommended in $\mathrm{CI}$ fittings for patients with bothersome tinnitus because a lower threshold setting may provide low-level background sound to mask the patient's tinnitus. Van de Heyning et al (2008) ${ }^{34}$ implemented this clinical approach by setting T-levels to 0 current units. The 22 patients in their study had unilateral hearing loss with severe tinnitus and received a standardlength $\mathrm{CI}$ for tinnitus relief. If the tinnitus percept was still present when T-levels were decreased to 0 current unit, then the levels were globally raised to $10 \%$ of the MCLs. The study revealed that fitting the unilateral $\mathrm{CI}$ in this manner was effective in reducing the patients' tinnitus loudness and handicap scores (Van de Heyning et al, 2008 ${ }^{34}$ ). Moreover, Liu et al $(2016)^{18}$ adjusted global T-levels using standard threshold measures for the $234 \mathrm{CI}$ patients included in their study. Liu et al (2016) ${ }^{18}$ reported that adjusting T-levels on electrodes that were pitch matched to the patient's tinnitus was not successful compared with a standard fitting procedure. No further details were provided. Finally, other groups have modified T-levels on individual electrodes on the array when stimulating the cochlea for tinnitus suppression (e.g., Rubinstein et al, $2003 ;{ }^{26}$ Zeng et al, $2011^{35}$ ). There appears to be many methods for adjusting T-levels for tinnitus suppression, and more research is needed to determine which approach is beneficial.

We have known for years that low-level background sounds and amplification from a hearing aid can be effective for many patients in reducing the prominence of tinnitus (e.g., Hazell et al, $1985^{13}$ ). Low-level background noise and amplified sound not only interferes with the tinnitus-related neural activity coded by the brain but also allows the listener to concentrate on external sounds instead of tinnitus.

\section{MCLs}

Several studies have shown that electrode current level influences tinnitus loudness. Rubinstein et al $(2003)^{26}$ found that higher current levels (equating to MCLs) were associated with rapid changes in tinnitus loudness for one CI patient whose tinnitus was suppressed with the conditioner stimulus. Higher current levels also elicited complete tinnitus suppression in many participants. Zeng et al $(2011)^{35}$ found a similar result where tinnitus was suppressed at higher current levels near the MCL for one CI patient. In that study, loudness scaling of the patient's tinnitus was completed by first obtaining a baseline measurement of the patient's tinnitus loudness, and then setting the MCL on selected electrodes for tinnitus suppression. Finally, Dauman and Tyler $(1993)^{4}$ showed that the current level needed to suppress tinnitus was also dependent on several factors, including the electrode position in the cochlea and the interelectrode distance. Specifically, less current was needed for tinnitus suppression when the electrode distance was greater (bipolar 13 versus bipolar 11; Dauman and Tyler, $1993^{4}$ ).

For some tinnitus patients, decreasing the MCL on certain electrodes (i.e., the basal electrodes that stimulate the cochlea at the same frequency of tinnitus) could reduce tinni- tus loudness. This might be effective for patients who experience changes to the pitch or quality of their tinnitus with CI use (Souliere et al, $1992^{28}$ ). These results on MCLs and tinnitus loudness are limited to a few participants, and more research from a greater number of $\mathrm{CI}$ patients with tinnitus is needed. In sum, the results currently suggest that the $\mathrm{CI}$ current level should be sufficient to suppress the patient's tinnitus, but not too high to cause loudness discomfort, pain, facial sensations, or other complications (Zeng et al, 2015 ${ }^{36}$ ).

\section{Possible Clinical Research Designs}

Given the lack of large-scale studies that systematically investigate $\mathrm{CI}$ programming for tinnitus suppression, we have considered possible research designs for clinical applications. First, in a daily alternating method, the audiologist fits two programs with different programming parameters and patients alternate daily between these programs to determine the optimal setting for tinnitus suppression. This strategy has been applied to $\mathrm{CI}$ and tinnitus patients and is a feasible method for selecting optimal programming parameters (Tyler et $\mathrm{al}, 2008 \mathrm{~b} ;{ }^{32} 2015^{33}$ ). Some patients required at least three months to determine their preference using the daily alternating method, and preferences changed over time from those at initial activation (Tyler et al, 2008b ${ }^{32}$ ).

Another method to evaluate the effectiveness of $\mathrm{CI}$ parameters for tinnitus suppression in a clinical setting is by changing only one parameter at a time during an at-home trial (see - Table 1). Audiologists start in step 1 by administering a pretrial tinnitus measure, such as a questionnaire or psychoacoustic measurement of tinnitus, to document the effectiveness of the current CI parameters in suppressing tinnitus. In step 2, the audiologist adjusts a single parameter in their $\mathrm{CI}$ program and provides the patient with an at-home trial for 2-3 months. We recommend changing only one single parameter at a time to isolate the effects of adjusting electrical stimulation for tinnitus suppression. The patient returns to the clinic after $2-3$ months, and at step 3, the same tinnitus measure would be re-administered after the trial to document the effectiveness of the new program in suppressing tinnitus. A speech recognition test is administered in steps 1 and 3 to ensure that speech perception is not significantly affected by the programming adjustments. In our studies, word recognition tests are presented at a normal conversational level (i.e., $60 \mathrm{~dB} \mathrm{HL}$ ) in the sound field with

Table 1 A Within-Subject Clinical Design for Evaluating the Effectiveness of $\mathrm{Cl}$ Programming Parameters for Tinnitus

\begin{tabular}{|l|l|l|}
\hline Step & Action & Description \\
\hline 1 & $\begin{array}{l}\text { Administer pretrial } \\
\text { tinnitus measure, } \\
\text { speech recognition test }\end{array}$ & $\begin{array}{l}\text { Document current } \\
\text { effectiveness of CI to } \\
\text { suppress tinnitus }\end{array}$ \\
\hline 2 & Adjust Cl programming & $\begin{array}{l}\text { At-home trial for } \\
\text { two months }\end{array}$ \\
\hline 3 & $\begin{array}{l}\text { Administer posttrial } \\
\text { tinnitus measure, } \\
\text { speech recognition test }\end{array}$ & $\begin{array}{l}\text { Document effectiveness } \\
\text { of new Cl programming } \\
\text { to suppress tinnitus }\end{array}$ \\
\hline
\end{tabular}


and without sound therapy. There are several advantages to these research designs alternating daily among programming parameters or providing an at-home trial with a new tinnitus suppression program. Specifically, these are withinsubject clinical research designs such that each patient serves as their own control and individual differences within the heterogeneous tinnitus population are considered.

Finally, the timing of the implementation of this protocol likely depends on each patient. For patients with severe tinnitus, this protocol may be appropriate to begin at initial activation, whereas if the tinnitus is not bothersome, a waiting period (e.g., after three months of CI use) may be warranted. Research that includes more participants with varying patient characteristics and tinnitus symptoms is needed to better understand which patients will benefit, when intervention should begin, and which parameters yield the best results for tinnitus suppression.

\section{Conclusions}

It is clear from the research literature that adjusting a $\mathrm{CI}$ to reduce the prominence of tinnitus is complex. The optimal stimulus parameters are likely to be different for different patients (Tyler et al, 2008a $\mathrm{a}^{31}$ ), and currently, the results are limited to a few participants across studies. There is no straightforward advice that can be given at this time for how to program a CI to suppress tinnitus. For patients with preoperative tinnitus that remains bothersome after implantation, the research suggests programming the $\mathrm{Cl}$ using a sophisticated signal coding strategy at high and low rates of stimulation and setting the current at a sufficient level to suppress tinnitus, but not causing discomfort. Then, the audiologist could consider adjusting the electrode number (e.g., eliminate or reactivate particular electrodes), T-levels (e.g., set at 0 versus measured Tlevels), andMCL adjustments at a later time using the proposed clinical research designs. Audiologists should explore some of these parameters for tinnitus suppression systematically when working with their $\mathrm{CI}$ patients. Single-participant experiments can be very helpful.

There are some $\mathrm{CI}$ patients who report tinnitus as a complication from CI surgery. In that group, tinnitus is largely experienced in the implanted ear and remains after surgery. A small portion of these patients experience improvements in their tinnitus once the $\mathrm{CI}$ is activated (Arts et al, 2015²). Therefore, if the patient presents with tinnitus after the surgery and it improves with $\mathrm{CI}$ use, there would be no need to further evaluate programming parameters for tinnitus suppression. However, an observation period may be beneficial to ensure that the tinnitus improves for patients who have tinnitus as a complication from $\mathrm{Cl}$ surgery. We suggest the following as next steps in the clinical management of $\mathrm{CI}$ patients with tinnitus:

- Document what audiologists are currently doing to manage their $\mathrm{CI}$ patients with tinnitus such as implementation of tinnitus management protocols, administration of questionnaires, testing with tinnitus psychoacoustic measurements, and counseling.
- Determine the successful approaches for managing tinnitus in $\mathrm{CI}$ patients, including $\mathrm{CI}$ programming parameters and sound therapy options.

- Develop a clinical protocol for programming CIs for tinnitus for busy clinics.

\section{Abbreviations}

\section{CI cochlear implant}

MCL most comfortable level

\section{Acknowledgments}

This work is supported by a tenure sabbatical leave grant awarded to Ann Perreau. Richard Tyler is a consultant for Cochlear Corporation.

\section{References}

1 Andersson G, Freijd A, Baguley DM, Idrizbegovic E. Tinnitus distress, anxiety, depression, and hearing problems among cochlear implant patients with tinnitus. J Am Acad Audiol 20;2009: 315-319

2 Arts RA, Netz T, Janssen AM, George EL, Stokroos RJ. The occurrence of tinnitus after $\mathrm{CI}$ surgery in patients with severe hearing loss: a retrospective study. Int J Audiol 54;2015;(12):910-917

3 Balkany T, Hodges A, Menapace C, Hazard L, Driscoll C, Gantz B, Kelsall D, Luxford W, McMenomy S, Neely JG, Peters B, Pillsbury H, Roberson J, Schramm D, Telian S, Waltzman S, Westerberg B, Payne S. Nucleus freedom north American clinical trial. Otolaryngol Head Neck Surg 136;2007:757-762

4 Dauman R, Tyler RS. Tinnitus suppression in cochlear implant users. Adv Otorhinolaryngol 48;1993:168-173

5 Dorman MF, Loizou PC, Kemp LL, Kirk KI. Word recognition by children listening to speech processed into a small number of channels: data from normal-hearing children and children with cochlear implants. Ear Hear 21;2000;(06):590-596

6 Firszt JB, Holden LK, Reeder RM, Skinner MW. Speech recognition in cochlear implant recipients: comparison of standard hires and hires 120 sound processing. Otol Neurotol 30;2009;(02):146-152

7 Fishman KE, Shannon RV, Slattery WH. Speech recognition as a function of the number of electrodes used in the SPEAK cochlear implant speech processor. J Speech Lang Hear Res 40;1997;(05): 1201-1215

8 Friesen LM, Shannon RV, Baskent D, Wang X. Speech recognition in noise as a function of the number of spectral channels: comparison of acoustic hearing and cochlear implants. J Acoust Soc Am 110;2001;(02):1150-1163

9 Frijns JHM, Klop WM, Bonnet RM, Briaire JJ, Briaire J. Optimizing the number of electrodes with high-rate stimulation of the clarion CII cochlear implant. Acta Otolaryngol 123;2003;(02):138-142

10 Gomersall PA, Baguley DM, Carlyon RP. A cross-sectional questionnaire study of tinnitus awareness and impact in a population of adult cochlear implant users. Ear Hear 40;2019:135-142

11 Hazell JWP, McKinney CJ, Aleksy W. Mechanisms of tinnitus in profound deafness. Ann Otol Rhinol Laryngol 1995 (Suppl 166):418-420

12 Hazell JWP, Meerton LJ, Conway MJ. 1989Electrical tinnitus suppression (ETS) with a single channel cochlear implant. J Laryngol Otol(Suppl 18):39-44

13 Hazell JW, Wood SM, Cooper HR, Stephens SDG, Corcoran AL, Coles RRA, Baskill JL, Sheldrake JB. A clinical study of tinnitus maskers. Br J Audiol 19;1985;(02):65-146

14 Ito J. Tinnitus suppression in cochlear implant patients. Otolaryngol Head Neck Surg 117;1997:701-703 
15 Koch DB, Osberger MJ, Segel P, Kessler D. HiResolution and conventional sound processing in the HiResolution bionic ear: using appropriate outcome measures to assess speech recognition ability. Audiol Neurootol 9;2004:214-223

16 Kou BS, Ship DB, Nedzelski JM. Subjective benefits reported by adult nucleus 22-channel cochlear implant users. J Otolaryngol 23;1994;(01):8-14

17 Kuk FK, Tyler RS, Rustad N, Harker LA, Tye-Murray N. Alternating current at the eardrum for tinnitus reduction. J Speech Hear Res 32;1989;(02):393-400

18 Liu Y, Wang H, Han DX, Li MH, Wang Y, Xiao YL. Suppression of tinnitus in Chinese patients receiving regular cochlear implant programming. Ann Otol Rhinol Laryngol 125;2016;(04):303-310

19 Mo B, Harris S, Lindbaek M. Tinnitus in cochlear implant patientsa comparison with other hearing-impaired patients. Int J Audiol 41;2002:527-534

20 Perreau A, Tyler RS, Witt SA. The effect of reducing the number of electrodes on spatial hearing tasks for bilateral cochlear implant recipients. J Am Acad Audiol 21;2010;(02):110-120

21 Plant KL, Whitford LA, Psarros CE, Vandali AE. Parameter selection and programming recommendations for the ACE and CIS speechprocessing strategies in the nucleus 24 cochlear implant system. Cochlear Implants Int 3;2002:104-125

22 Punte AK, De Ridder D, Van de Heyning P. On the necessity of full length electrical cochlear stimulation to suppress severe tinnitus in single-sided deafness. Hear Res 295;2013:24-29

23 Quaranta N, Wagstaff S, Baguley DM. Tinnitus and cochlear implantation. Int J Audiol 43;2004:245-251, Review paper

24 Quaranta N, Fernandez-Vega S, D'Elia C, Filipo R, Quaranta A. The effect of unilateral multichannel cochlear implant on bilaterally perceived tinnitus. Acta Otolaryngol 128;2008:159-163

25 Rubinstein JT, Tyler RS. Electrical suppression of tinnitus. In: Snow J, ed. Tinnitus: Theory and Management. Hamilton, Canada: B.C. Decker; 326-335
26 Rubinstein JT, Tyler RS, Johnson A, Brown CJ. Electrical suppression of tinnitus with high-rate pulse trains. Otol Neurotol 24;2003: 478-485

27 Schvartz-Leyzac KC, Zwolan TA, Pfingst BE. Effects of electrode deactivation on speech recognition in multichannel cochlear implant recipients. Cochlear Implants Int 18;2017;(06):324-334

28 Souliere CR JR, Kileny PR, Zwolan TA, Kemink JL. Tinnitus suppression following cochlear implantation. A multifactorial investigation. Arch Otolaryngol Head Neck Surg 118;1992;(12):1291-1297

29 Tyler RS. Advantages and disadvantages expected and reported by cochlear implant patients. Am J Otol 15;1994:523-531

30 Tyler RS, Kelsay D. Advantages and disadvantages reported by some of the better cochlear-implant patients. Am J Otol 11;1990: 282-289

31 Tyler RS, Rubinstein J, Pan T, Chang S, Gogel S, Gehringer A, Coelho C. Electrical stimulation of the cochlea to reduce tinnitus. Semin Hear 29;2008a(04):326-332

32 Tyler RS, Witt S, Dunn CC, Perreau A. A daily alternating method for comparing different signal-processing strategies in hearing aids and in cochlear implants. J Am Acad Audiol 19;2008b443-454

33 Tyler RS, Keiner AJ, Walker K, Deshpande AK, Witt S, Killian M, Ji H, Patrick J, Dillier N, van Dijk P, Lai WK, Hansen MR, Gantz B. A series of case studies of tinnitus suppression with mixed background stimuli in a cochlear implant. Am J Audiol 22;2015:398-410

34 Van de Heyning P, Vermeire K, Diebl M, Nopp P, Anderson I, De Ridder D. Incapacitating unilateral tinnitus in single-sided deafness treated by cochlear implantation. Ann Otol Rhinol Laryngol 117;2008:645-652

35 Zeng FG, Tang Q, Dimitrijevic A, Starr A, Larky J, Blevins NH. Tinnitus suppression by low-rate electric stimulation and its electrophysiological mechanisms. Hear Res 277;2011:61-66

36 Zeng FG, Djalilian H, Lin H. Tinnitus treatment with precise and optimal electric stimulation: opportunities and challenges. Curr Opin Otolaryngol Head Neck Surg 23;2015;(05):382-387 